\title{
Potencial de infectividade de fungos micorrízicos arbusculares em áreas nativas e impactadas por mineração gesseira no semi-árido brasileiro
}

\author{
Adália Cavalcanti do Espírito Santo Mergulhão ${ }^{1,3}$, José de Paula Oliveira ${ }^{1}$, Hélio Almeida Burity ${ }^{1}$ e \\ Leonor Costa Maia $^{2}$
}

Recebido: 23.11.2006; aceito: 05.07.2007

ABSTRACT - (Infectivity potential of arbuscular mycorrhizal fungi in native and impacted gypsum mining areas of the Brazilian semi-arid). The effect of gypsum mining on arbuscular mycorrhizal fungi (AMF) was evaluated. Soil samples (10/area) were collected in four areas of a mine: native "caatinga" $(\mathrm{CN})$; mine surroundings (AM); waste deposit (R) and interface between waste deposit and degraded caatinga (IN), in two periods, raining (December/2003) and dry (September/2004). Fourty two phanerogam species were registered: 25 at $\mathrm{CN}, 14$ at AM, 10 at IN, and 5 at R. The CN presented higher number of plant species, spores and infectives propagules (most probable number-MPN) of AMF. Number of spores and root colonization differed between the periods. Higher sporulation occurred in the raining season, MPN of propagules and colonization were higher in the dry season. Positive correlation occurred between MPN and spores in both periods. In the raining season, MPN was negatively correlated with colonization. That was higher in the AM and R areas. The mining activity decreased plant diversity, MPN of propagules and amount of AMF spores in relation to the native caatinga.

Key words: arbuscular mycorrhizae fungi, infective propagules, root colonization

RESUMO - (Potencial de infectividade de fungos micorrízicos arbusculares em áreas nativas e impactadas por mineração gesseira no semi-árido brasileiro). Foi avaliado o efeito da mineração gesseira sobre os fungos micorrízicos arbusculares (FMA). Amostras de solo (10/área) foram coletadas em quatro áreas de uma mineradora: caatinga nativa (CN), arredores da mina (AM), rejeito (R) e interface entre o rejeito e caatinga degradada (IN), nos períodos chuvoso (dezembro/2003) e seco (setembro/2004). Foram registradas 42 espécies de fanerógamas: 25 na CN, 14 nos AM, 10 na IN e cinco na R. A CN apresentou maior número de espécies vegetais, esporos e propágulos infectivos de FMA. Número de esporos e colonização radicular diferiram entre os períodos. Maior esporulação ocorreu no período chuvoso, o número mais provável (NMP) de propágulos e colonização foram maiores no seco. Houve correlação positiva entre NMP e esporos nos dois períodos. No período chuvoso, o NMP foi negativamente correlacionado com colonização. Esta foi maior nas áreas AM e R. A atividade mineradora diminuiu a diversidade vegetal, o NMP de propágulos e a quantidade de esporos em relação à caatinga nativa. Palavras-chave: colonização radicular, fungos micorrízicos arbusculares, propágulos infectivos

\section{Introdução}

Os fungos micorrízicos arbusculares (FMA) são importantes componentes da microbiota do solo em ecossistemas naturais e agrícolas, formando associação endomicorrízica com a maioria das plantas superiores incluindo quase todas as espécies de interesse agronômico, pastoril e florestais tropicais (Moreira \& Siqueira 2002). A atividade de mineração exerce impacto sobre os organismos e processos do solo, inclusive sobre os FMA (Melloni et al. 2003). Gould et al. (1996) observaram que o decréscimo nas populações de FMA em solo impactado poderia estar relacionado à perturbação física e ao armazenamento inadequado do solo superficial quando transferido para outra área, provocando a redução da viabilidade dos esporos e o rompimento da rede de hifas no solo. A perturbação do solo causada pelas atividades de mineração prejudica a comunicação das plantas com seus parceiros micorrízicos. Por outro lado, a remoção

1. Empresa Pernambucana de Pesquisa Agropecuária, Laboratórios de Biologia do Solo e Genoma, Av. General San Martin 1371, 50761-000 Recife, PE, Brasil

2. Universidade Federal de Pernambuco, Departamento de Micologia, Laboratório de Micorriza, Av. Prof. Nelson Chaves s.n., 50670-420 Cidade Universitária, Recife, PE, Brasil

3. Autor para correspondência: adalia@ipa.br 
do solo superficial pode deixar poucos propágulos de FMA capazes de iniciar a colonização micorrízica, influenciando a diversidade desses fungos durante o estádio inicial de recuperação da área impactada e, em certos casos, até mesmo impedindo o estabelecimento de plantas micorriza-dependentes (Loree \& Williams 1987), alterando a diversidade vegetal.

Observações no padrão de sucessão de plantas em regiões semi-áridas indicam que os FMA desempenham importante papel ecológico na composição e estabilidade das comunidades de plantas (Marx 2000). Em geral a inoculação com FMA melhora a produtividade das plantas pelo aumento da tolerância à seca (essencialmente em regiões áridas) e da disponibilidade de minerais, os primeiros fatores limitantes na estabilidade das plantas em solos sob atividade mineradora (Marx 2000). Segundo Jasper et al. (1994), nível adequado de infectividade de FMA no solo, após o processo de mineração, é importante para o sucesso do restabelecimento da diversidade vegetal na formação de um ecossistema auto-sustentável.

As características químicas e físicas dos solos afetam a densidade de esporos, a composição de espécies e a distribuição dos FMA, influenciando diferentemente o crescimento das plantas (Day et al. 1987). Segundo Brundrett \& Abbott (1994), o potencial de infectividade dos FMA é a capacidade de seus propágulos em colonizar as raízes de plantas, sendo mensurado pela percentagem de colonização nas raízes de uma planta hospedeira. Existem no solo três tipos de propágulos de FMA capazes de originar a simbiose: esporos de resistência, fragmentos de raiz micorrizada e hifas do fungo, os quais diferem quanto à capacidade de sobrevivência e capacidade de iniciar a colonização (Silveira 1992). O potencial de infectividade pode ser estimado pela técnica do número mais provável (NMP), a qual enumera todos os propágulos que têm habilidade para colonizar a planta hospedeira (Sieverding 1991). Outros parâmetros utilizados para estudos das populações de FMA no solo são a contagem de esporos e a identificação taxonômica das espécies.

Estudos sobre FMA em ecossistemas degradados por atividade mineradora são escassos no Brasil. Algumas pesquisas foram realizadas em área de mineração de bauxita no Pará (Caproni et al. 2003, 2005, Melloni et al. 2003) e na Amazônia (Marinho et al. 2004) e em área de mineração de cobre na Bahia (Silva et al. 2001a, 2005). Não foram encontradas referências sobre FMA em áreas impactadas pela mineração de gesso no país, sendo importante avaliar os efeitos dessa atividade sobre esses simbiontes. O objetivo desse trabalho foi testar a hipótese de que os FMA são afetados negativamente pela exploração do solo em área com mineração de gesso.

\section{Material e métodos}

O estudo foi conduzido em áreas de caatinga nativa preservada e impactada pela mineração de gipsita no município de Araripina, PE ( $7^{\circ} 29^{\prime} 00^{\prime}$ 'S e $40^{\circ} 36^{\prime} 00^{\prime}$ W). A região apresenta clima Semi-árido mesotérmico, a vegetação predominante é caatinga hiperxerófila e o solo do tipo Latossolo vermelho amarelo distrófico (Cavalcanti \& Lopes 1994).

As coletas foram realizadas em quatro áreas da mineradora: caatinga nativa preservada $(\mathrm{CN})$, considerada como controle, arredores da mina (AM), rejeito $(\mathrm{R})$ e na interface entre o depósito de rejeito e uma área de caatinga degradada pela mineração (IN). As áreas foram amostradas no período chuvoso $($ dezembro/2003, precipitação $=28,7 \mathrm{~mm}$ ) e seco (setembro/2004, precipitação =1,3 mm). A precipitação acumulada nos dois meses anteriores e no da coleta no período chuvoso (outubro/novembro/dezembro/ 2003) chegou a $41,9 \mathrm{~mm}$, atingindo apenas $4,3 \mathrm{~mm}$ nos meses correspondentes ao período seco (julho/ agosto/setembro/2004). Esses dados de precipitação foram obtidos junto a Empresa Pernambucana de Pesquisa Agropecuária (Tavares, dados não publicados). Para coleta foi definida como parâmetro uma área de $1.000 \mathrm{~m}^{2}$, sendo coletadas nas rizosferas a cada $10 \mathrm{~m}$, em zig-zag, dez amostras compostas de três subamostras de solo tiradas de 5 a $20 \mathrm{~cm}$ de profundidade. Em cada ponto de coleta do solo foi considerado um raio de $2 \mathrm{~m}$, onde foram coletadas amostras das plantas ocorrentes que foram identificadas no Laboratório de Botânica da Empresa Pernambucana de Pesquisa Agropecuária (IPA). Amostras de solo também foram encaminhadas ao IPA para análises físicas, químicas e de fertilidade (tabela 1).

Os esporos de FMA foram extraídos do solo por peneiramento úmido (Gerdemann \& Nicolson 1963), centrifugação em água e sacarose (Jenkins 1964) e contados em microscópio estereoscópico. As raízes retiradas das amostras de solo rizosférico foram diafanizadas e coradas (Phillips \& Hayman 1970) para avaliação da colonização micorrízica. Esta foi estimada em 200 fragmentos de $1 \mathrm{~cm}$ de raiz/amostra, 
Tabela 1. Características químicas e físicas do solo coletado nos períodos chuvoso (dezembro/2003) e seco (setembro/2004), em quatro áreas de mineradora de gipsita, em Araripina, PE. ${ }^{1} \mathrm{CN}=$ Caatinga nativa preservada; $\mathrm{IN}=$ interface entre depósito de rejeito e área de caatinga degradada pela mineração; $\mathrm{AM}=$ arredores da mina; $\mathrm{R}=$ rejeito.

\begin{tabular}{|c|c|c|c|c|c|c|c|c|c|c|c|c|}
\hline \multirow[t]{2}{*}{ Áreas ${ }^{1}$} & \multirow{2}{*}{$\begin{array}{r}\mathrm{pH} \\
\mathrm{H}_{2} \mathrm{O}\end{array}$} & \multirow{2}{*}{$\begin{array}{c}\mathrm{P} \\
\mathrm{mg} \mathrm{dm} \mathrm{dm}^{-3}\end{array}$} & \multirow{2}{*}{$\begin{array}{c}\mathrm{C} \\
\mathrm{g} / \mathrm{kg}\end{array}$} & \multirow{2}{*}{\multicolumn{3}{|c|}{$\begin{array}{lc}\mathrm{Ca} \quad \mathrm{Mg} \\
& \mathrm{cmolc} \mathrm{dm}^{-3}\end{array}$}} & \multirow[t]{2}{*}{$\mathrm{SO}_{4}$} & \multirow{2}{*}{$\frac{\mathrm{CO}_{3}}{\mathrm{meq} / \mathrm{L}}$} & \multirow[t]{2}{*}{$\mathrm{HCO}_{3}$} & \multicolumn{3}{|c|}{ Análise Textural (\%) } \\
\hline & & & & & & & & & & Areia & Argila & Silte \\
\hline \multicolumn{13}{|c|}{ Período Chuvoso (dezembro/2003) } \\
\hline $\mathrm{CN}$ & 6,28 & 5 & 1,16 & 5,90 & 1,30 & 0,43 & Presença & 0,20 & 1,80 & 67 & 8 & 25 \\
\hline IN & 5,72 & 11 & 1,27 & 20,00 & 9,30 & 0,46 & Presença & 0,20 & 0,80 & 26 & 45 & 29 \\
\hline $\mathrm{AM}$ & 7,64 & 54 & 1,34 & 40,50 & 6,25 & 0,19 & Forte Presença & 0,40 & 1,60 & 22 & 39 & 39 \\
\hline $\mathrm{R}$ & 7,65 & 42 & 0,72 & 68,00 & 9,75 & 0,34 & Forte Presença & 0,20 & 1,20 & 27 & 6 & 67 \\
\hline \multicolumn{13}{|c|}{ Período Seco (setembro/2004) } \\
\hline $\mathrm{CN}$ & 6,43 & 8 & 1,20 & 6,20 & 1,15 & 0,36 & Presença & 0,80 & 5,60 & 74 & 8 & 18 \\
\hline IN & 5,72 & 14 & 1,30 & 36,25 & 11,25 & 0,62 & Forte Presença & 0,00 & 0,80 & 14 & 59 & 27 \\
\hline $\mathrm{AM}$ & 7,52 & 161 & 1,33 & 45,40 & 3,10 & 0,40 & Forte Presença & 0,00 & 1,60 & 22 & 47 & 31 \\
\hline $\mathrm{R}$ & 7,56 & 32 & 0,65 & 61,75 & 1,65 & 0,46 & Forte Presença & 0,20 & 2,40 & 37 & 28 & 35 \\
\hline
\end{tabular}

sendo considerada colonizada a que apresentava arbúsculos, vesículas, esporos e/ou micélio.

O número mais provável (NMP) de propágulos infectivos de FMA foi calculado pela técnica descrita em Feldmann \& Idczak (1992). Bioensaios em casade-vegetação foram montados para cada área e para cada período de coleta. A temperatura e a umidade relativa do ar foram medidas diariamente, variando de 21 a $35{ }^{\circ} \mathrm{C}$ e de 31 a $88 \%$, respectivamente. Para cada área (CN, IN, AM e R) foi usada uma amostra composta de solo do campo, homogeneizado, seco, não esterilizado e peneirado (malha de $0,5 \mathrm{~cm}$ de abertura), sendo esta a amostra base para as diluições (solo-inóculo). O solo diluente (correspondente à amostra composta de cada área) foi homogeneizado, peneirado (malha de $0,5 \mathrm{~cm}$ de abertura), autoclavado por $1 \mathrm{~h}$ a $120^{\circ} \mathrm{C}$ por três dias consecutivos e seco em estufa a $105^{\circ} \mathrm{C}$. As amostras do solo-inóculo foram diluídas nas proporções de $0,1: 10,1: 100$ e 1:1.000 (solo-inóculo:solo-diluente, v:v) e colocadas em potes plásticos de $400 \mathrm{~mL}$, com cinco repetições, totalizando 160 unidades. Cada pote recebeu duas sementes de milho (Zea mays L.) e após a germinação ( \pm 5 dias) apenas uma plântula foi mantida. As plantas foram coletadas aos 30 dias e todo o sistema radicular preparado para verificação da presença de estruturas de FMA. O NMP de propágulos $\mathrm{g}^{-1}$ de solo amostrado foi obtido na tabela de Cochran (1950).

A densidade total de esporos e o percentual de colonização radicular foram submetidos à análise de variância (ANOVA) considerando os dez pontos amostrais em cada área como repetições. As médias das diferentes áreas e de períodos de avaliação foram comparadas pelo teste de Tukey $(\mathrm{P} \leq 0,05)$ utilizando o programa Sanest (Zonta et al. 1984). O número de esporos foi transformado em $\log (X+1,5)$, e a percentagem de colonização radicular em $\sqrt{(X+0,5)}$ para minimizar a variância. Foram realizadas análises de correlação simples de Pearson (r) entre as variáveis estudadas: número de esporos, NMP de propágulos infectivos e colonização radicular (Stasoft 1997).

\section{Resultados e Discussão}

Foram identificadas 42 espécies, representando 25 famílias de fanerógamas, considerando todos os pontos de coleta e os dois períodos. As famílias mais bem representadas foram Leguminosae (Mimosoidae) e Euphorbiaceae, com seis e quatro espécies, respectivamente. A atividade mineradora causou danos ao local, diminuindo a diversidade vegetal nas três áreas afetadas pela mineração quando comparadas com a área preservada. Assim, enquanto na área nativa registraram-se 25 espécies, nos arredores esse número diminuiu para 14, na interface dez e na área de rejeito apenas cinco espécies foram reconhecidas (tabela 2). Vinte espécies foram encontradas exclusivamente na área nativa, enquanto 17 foram observadas apenas nas áreas impactadas. Silva et al. (2005) verificaram que a atividade mineradora para exploração de cobre no semi-árido nordestino também reduziu o número de espécies vegetais quando comparado ao registrado em área nativa. Em relação à comunidade de FMA, o número de esporos e de propágulos infectivos igualmente foi maior na área preservada comparado às outras áreas (tabelas 3-4). 
Tabela 2. Espécies vegetais registradas em quatro áreas de mineradora de gipsita, em Araripina, $\mathrm{PE}$. $\mathrm{CN}=$ caatinga nativa preservada; $\mathrm{AM}=$ arredores da mina; $\mathrm{R}=$ rejeito; $\mathrm{IN}$ = interface entre depósito de rejeito e área de caatinga degradada pela mineração.

\begin{tabular}{|c|c|c|c|c|c|}
\hline \multirow[t]{2}{*}{ Família/Espécie } & \multirow[t]{2}{*}{ Nome vulgar } & \multicolumn{4}{|c|}{ Áreas } \\
\hline & & $\mathrm{CN}$ & $\mathrm{AM}$ & $\mathrm{R}$ & IN \\
\hline \multicolumn{6}{|l|}{ ACANTHACEAE } \\
\hline Ruellia paniculata L. & - & & $\mathrm{X}$ & $\mathrm{X}$ & \\
\hline Dicliptera sp. & - & $\mathrm{X}$ & & & \\
\hline \multicolumn{6}{|l|}{ AMARANTHACEAE } \\
\hline Alternanthera tenella Colla & Ervanso & & $\mathrm{X}$ & $\mathrm{X}$ & \\
\hline \multicolumn{6}{|l|}{ ANACARDIACEAE } \\
\hline Myracrodruon urundeuva Alemão & Aroeira & $\mathrm{X}$ & & & \\
\hline Spondias tuberosa Arruda & Umbuzeiro & $\mathrm{X}$ & & & \\
\hline \multicolumn{6}{|l|}{ ANNONACEAE } \\
\hline Rollinia leptopetala R.E. Fries & Bananinha & $\mathrm{X}$ & & & \\
\hline \multicolumn{6}{|l|}{ APOCYNACEAE } \\
\hline Aspidosperma pyrifolium Mart. & Pereiro & $\mathrm{X}$ & & & $\mathrm{X}$ \\
\hline \multicolumn{6}{|l|}{ ASTERACEAE } \\
\hline Tagetes minuta $\mathrm{L}$. & Cravo-branco & & $\mathrm{X}$ & $\mathrm{X}$ & \\
\hline \multicolumn{6}{|l|}{ BIGNONIACEAE } \\
\hline Arrabidaea corallina (Jacq.) Sandw. & Cipó de balaio & & $\mathrm{X}$ & & \\
\hline \multicolumn{6}{|l|}{ BORAGINACEAE } \\
\hline Heliotropium procumbens Mill. & Borragem & & & & $\mathrm{X}$ \\
\hline \multicolumn{6}{|l|}{ BROMELIACEAE } \\
\hline Tillandsia loliacea Mart. & - & $\mathrm{X}$ & & & \\
\hline \multicolumn{6}{|l|}{ BURSERACEAE } \\
\hline Commiphora leptophloeos (Mart.) Gillet. & Umburana & $\mathrm{X}$ & & & \\
\hline \multicolumn{6}{|l|}{ CACTACEAE } \\
\hline \multirow{2}{*}{\multicolumn{6}{|c|}{ CARICACEAE }} \\
\hline & & & & & \\
\hline Jacaratia sp. & Mamãozinho & $\mathrm{X}$ & & & \\
\hline \multicolumn{6}{|l|}{ CAPPARACEAE } \\
\hline Caparis hastata $\mathrm{L}$. & Feijão-bravo & $\mathrm{X}$ & & & \\
\hline Caparis flexuosa $\mathrm{L}$. & Feijão-de-boi & $\mathrm{X}$ & $\mathrm{X}$ & & \\
\hline \multicolumn{6}{|l|}{ CONVOLVULACEAE } \\
\hline Iротоеа sp. & Jitirana & $\mathrm{X}$ & & & \\
\hline Ipomoea phyllomega (Vell.) House & - & $\mathrm{X}$ & & & \\
\hline Merremia sp. & - & & $\mathrm{X}$ & & \\
\hline \multicolumn{6}{|l|}{ EUPHORBIACEAE } \\
\hline Croton sonderianus Mull. Arg. & Marmeleiro-preto & $\mathrm{X}$ & & & \\
\hline Croton sp. & Marmeleiro & $\mathrm{X}$ & $\mathrm{X}$ & & $\mathrm{X}$ \\
\hline Manihot sp. & Maniçoba & & & & $\mathrm{X}$ \\
\hline Jatropha mollissima (Pohl.) Baill. & Pinhão-bravo & & $\mathrm{X}$ & & \\
\hline \multicolumn{6}{|l|}{ LEGUMINOSAE CAESALPINOIDEAE } \\
\hline Bauhinia cheilantha Steud. & Mororó & $\mathrm{X}$ & & & \\
\hline Cassia trachypus Mart. & Canafístula & & $X$ & & \\
\hline LEGUMINOSAE MIMOSOIDAE & & & & & \\
\hline Parapiptadenia zehntneri (Harms) M.P. Lima & Angico-branco & $\mathrm{X}$ & & & \\
\hline Mimosa tenuiflora (Willd.) Poir. & Jurema-preta & $\mathrm{X}$ & & & \\
\hline Mimosa ophthalmocentra Mart. & Jurema de embira & $\mathrm{X}$ & $\mathrm{X}$ & & \\
\hline Anadenanthera colubrina (Vell.) Brenan. & Angico-de-caroço & $\mathrm{X}$ & & & $\mathrm{X}$ \\
\hline Prosopis juliflora DC. & Algaroba & & $\mathrm{X}$ & $\mathrm{X}$ & $\mathrm{X}$ \\
\hline Desmanthus virgatus (L.) Willd. & Anis-de-bode & & $\mathrm{X}$ & & \\
\hline LEGUMINOSAE PAPILIONOIDEAE & & & & & \\
\hline Rhynchosia minima (L.) DC. & Feijãozinho-de-capoeira & & & & $\mathrm{X}$ \\
\hline Canavalia sp. & - & $\mathrm{X}$ & & & \\
\hline MALPHIGIACEAE & & & & & \\
\hline Banisteria cotinifolia A. Juss. & - & $\mathrm{X}$ & & & \\
\hline
\end{tabular}


Tabela 2 (continuação)

\begin{tabular}{|c|c|c|c|c|c|}
\hline \multirow[t]{2}{*}{ Família/Espécie } & \multirow[t]{2}{*}{ Nome vulgar } & \multicolumn{4}{|c|}{ Áreas } \\
\hline & & $\mathrm{CN}$ & $\mathrm{AM}$ & $\mathrm{R}$ & IN \\
\hline \multicolumn{6}{|l|}{ MALVACEAE } \\
\hline Sida sp. & Malva & & $\mathrm{X}$ & & \\
\hline Sida cordifolia $\mathrm{L}$. & Malva-grossa & & & $\mathrm{X}$ & \\
\hline Sidastrum micranthum (A. St. Hill.) Fryxell & Malva-preta & & & & $\mathrm{X}$ \\
\hline \multicolumn{6}{|l|}{ NYCTAGINACEAE } \\
\hline Guapira noxia (Netto) Sundell. & Piranha & $\mathrm{X}$ & & & \\
\hline \multicolumn{6}{|l|}{ RHAMNACEAE } \\
\hline Zizyphus joazeiro Mart. & Juazeiro & & $\mathrm{X}$ & & $\mathrm{X}$ \\
\hline \multicolumn{6}{|l|}{ SAPINDACEAE } \\
\hline Allophylus quercifolia (Mart.) Radlk. & - & $\mathrm{X}$ & & & \\
\hline \multicolumn{6}{|l|}{ SELAGINELLACEAE } \\
\hline Sellaginella convoluta Spring. & Jericó & $\mathrm{X}$ & & & \\
\hline \multicolumn{6}{|l|}{ VITACEAE } \\
\hline Cissus simsiana Schult \& Schult & Parreira-brava & $\mathrm{X}$ & & & \\
\hline Total & & 25 & 14 & 5 & 10 \\
\hline
\end{tabular}

Como a esporulação é dependente da formação de novas raízes (Sieverding 1991), provavelmente na área nativa $(\mathrm{CN})$, onde as condições ambientais são mais adequadas e não se observa impacto ambiental, a riqueza de espécies vegetais pode ter estimulado o aumento da população de FMA.

A quantidade de esporos na área nativa foi significativamente maior (média de 4,2 esporos) do que nas demais áreas. Em área de caatinga explorada por mineração de cobre, na Bahia, o número de esporos de FMA foi sempre inferior a $1,6 \mathrm{~g}^{-1}$ de solo, mesmo na área nativa (Silva et al. 2001a). Verificou-se, no período chuvoso, maior produção de esporos (média 3 esporos $\mathrm{g}^{-1}$ de solo) mostrado na tabela 4. Esses dados coincidem com os de Caproni et al. (2005), que verificaram aumento na densidade de esporos no período chuvoso, em áreas revegetadas com Acacia mangium Willd após mineração de bauxita na região de Porto Trombetas, PA. O mesmo foi observado por Coelho et al. (1997) em solo sob plantio de Eucalyptus spp., em São Paulo. A ocorrência de variação sazonal na dinâmica micorrízica foi proposta por Allen et al. (1998); no entanto, nem sempre tais diferenças são registradas, com o número de esporos mantendo-se similar nas diferentes estações do ano, em condições semi-áridas (Silva et al. 2001a).

Houve efeito isolado dos fatores (áreas e períodos de amostragem), mas sem interação para o número de esporos (áreas $\mathrm{P}<0,0001$; períodos de amostragem $\mathrm{P}<0,0001)$ e percentual de colonização radicular (áreas $\mathrm{P}<0,0002$; períodos de amostragem
$\mathrm{P}<0,0001)$. Isso foi observado, sobretudo, na área CN (tabela 4). Louis \& Lim (1987) também verificaram, em solos tropicais de floresta úmida, que quando o número de esporos era alto, a percentagem de colonização era baixa e vice-versa. Os autores sugeriram que a variação nos percentuais de colonização pode ser influenciada pela interação entre o estado nutricional dos solos tropicais, a planta hospedeira e o ciclo de vida dos FMA.

Verificou-se maior NMP de propágulos infectivos de FMA durante o período com pouca chuva, em todas as áreas estudadas (tabela 3). É possível que o aumento da umidade no período chuvoso favoreça a germinação de esporos mas, não encontrando hospedeiro susceptível, os fungos acabem morrendo.

Tabela 3. Número mais provável (NMP) de propágulos de FMA em áreas de caatinga nativa e impactada pela mineração de gipsita, em dois períodos de coleta (chuvoso = dezembro/2003; seco $=$ setetembro/2004) em Araripina, PE. *Entre parênteses limite de confiança mínimo e máximo a $95 \%$ de probabilidade (Cochran 1950). $\mathrm{CN}=$ caatinga nativa preservada; $\mathrm{AM}=$ arredores da mina; $\mathrm{R}=$ rejeito; $\mathrm{IN}$ = interface entre depósito de rejeito e área de caatinga degradada pela mineração.

\begin{tabular}{ccc}
\hline \multirow{2}{*}{ Áreas } & \multicolumn{2}{c}{ NMP de propágulos $\mathrm{g}^{-1}$ de solo } \\
\cline { 2 - 3 } & Período chuvoso & Período seco \\
\hline $\mathrm{CN}$ & $170(52-561)^{*}$ & $540(164-1.782)$ \\
$\mathrm{IN}$ & $26(8-86)$ & $180(55-594)$ \\
$\mathrm{AM}$ & $22(7-73)$ & $140(42-462)$ \\
$\mathrm{R}$ & $17(5-56)$ & $130(39-429)$ \\
\hline
\end{tabular}


Tabela 4. Colonização radicular e número de esporos de FMA em quatro áreas de mineração de gipsita, em Araripina, PE, considerando áreas e períodos de coleta. $\mathrm{CN}=$ caatinga nativa preserva$\mathrm{da} ; \mathrm{AM}=$ arredores da mina; $\mathrm{R}=$ rejeito; $\mathrm{IN}=$ interface entre depósito de rejeito e área de caatinga degradada pela mineração. Médias seguidas da mesma letra, na coluna, não diferem significativamente pelo teste de Tukey $(\mathrm{P}<0,05)$ * Médias originais, transformadas em $\sqrt{(\mathrm{X}+0,5)}$ para a análise de variância, $\mathrm{CV}$ $(\%)=23$. ${ }^{*}$ Médias originais, transformadas em $\log (\mathrm{X}+1,5)$ para a análise de variância, CV $(\%)=30$.

\begin{tabular}{lcc}
\hline & $\begin{array}{c}\text { Colonização } \\
\text { radicular (\%)* }\end{array}$ & $\begin{array}{c}\text { Número de } \\
\text { esporos } \mathrm{g}^{-1} \text { de solo** }\end{array}$ \\
\hline Áreas & & \\
$\mathrm{CN}$ & $37,5 \mathrm{~b}$ & $4,2 \mathrm{a}$ \\
$\mathrm{IN}$ & $42,5 \mathrm{~b}$ & $0,7 \mathrm{~b}$ \\
$\mathrm{AM}$ & $55,0 \mathrm{a}$ & $1,2 \mathrm{~b}$ \\
$\mathrm{R}$ & $56,8 \mathrm{a}$ & $1,4 \mathrm{~b}$ \\
Períodos & & \\
Chuvoso (dezembro/2003) & $26,4 \mathrm{~b}$ & $3,0 \mathrm{a}$ \\
Seco (setembro/2004) & $69,5 \mathrm{a}$ & $0,7 \mathrm{~b}$ \\
\hline
\end{tabular}

Esta hipótese poderia explicar os menores valores de propágulos infectivos em todas as áreas, sobretudo naquelas impactadas, durante o período com maior precipitação. Por outro lado, a técnica do NMP pode ativar alguns propágulos que nas condições de campo não seriam infectivos (Abbott \& Robson 1991).

Enquanto maior esporulação foi verificada no período chuvoso (tabela 4), o NMP de propágulos infectivos foi menor neste período (tabela 3). No caso, houve correlação positiva entre o NMP de propágulos infectivos de FMA e o número total de esporos no solo nos dois períodos avaliados (chuvoso $=0,98 \mathrm{e}$ seco $=0,95)$. Porém, nem sempre tal correlação é observada (Sieverding 1991). O potencial de inóculo de um solo, que está relacionado a fragmentos de raízes micorrizadas e ao micélio fúngico, pode também estar relacionado ao número de esporos presentes no ambiente (Collozi-Filho \& Balota 1994). Portanto, apesar da quantidade de esporos no solo não fornecer o valor real de sua infectividade, pode dar indicação do nível populacional dos FMA (Dodd et al. 1983).

De modo geral o NMP de propágulos infectivos de FMA foi sempre superior ao número de esporos no solo (tabelas 3-4). Dado contrário foi registrado por Silva et al. (2001a), os quais observaram que o número de esporos no solo era sempre maior do que o de propágulos infectivos. An et al. (1990) compararam os métodos do NMP e o de contagem direta de esporos e concluíram que o número de esporos pode ser menor ou maior que o de propágulos infectivos, em função da espécie de FMA.

A análise do solo revelou elevados teores de fósforo, cálcio e magnésio nos dois períodos de amostragem nas áreas impactadas pela mineração (tabela 1). O aumento do nível de fósforo no solo pode influenciar espécies de FMA hábeis em colonizar as raízes das plantas (Abbott \& Robson 1991), tal como registrado por Douds Júnior (1994), o qual observou diminuição na produção de esporos de FMA com o incremento de P no solo. Gould et al. (1996) observaram que o teor de $\mathrm{Mg}$ foi inversamente relacionado com a ocorrência de esporos de FMA em áreas de mineração e revegetadas. Por outro lado, há registro do efeito negativo de altas doses de Ca no solo sobre a germinação de esporos de FMA (Siqueira et al. 1986). É difícil separar os fatores mais importantes na interação micorrízica, embora no campo as concentrações de propágulos de FMA sejam correlacionadas, principalmente, com as condições do solo e a cobertura vegetal (Sieverding 1991). Ainda segundo esse autor, os menores números de propágulos de FMA são encontrados em áreas com vegetação degradada.

Correlação negativa significativa foi verificada entre o NMP e a colonização radicular $(-0,98)$ durante o período chuvoso, enquanto no período seco esses dois parâmetros não se correlacionaram $(-0,66 \mathrm{~ns})$. As áreas de $\mathrm{CN}$ e de IN apresentaram menor percentual de colonização radicular, embora houvesse número considerável de propágulos infectivos no solo, sobretudo na área $\mathrm{CN}$ (tabelas 3-4).

A colonização radicular variou de 37,5 a $56,8 \%$ entre as áreas estudadas, sendo maior naquelas ( $\mathrm{AM}=55 \%$ e $\mathrm{R}=56,8 \%)$ sob forte impacto ambiental (tabela 4). Em raízes de Commelinidae coletadas em áreas de caatinga, no Estado de Pernambuco, Silva et al. (2001b) registraram percentual de colonização radicular que variou de 4 a 50\%. A micorrização de plantas da caatinga pode estar relacionada a diversos fatores que interferem na colonização, tais como: susceptibilidade e competitividade nutricional dos hospedeiros, micotrofismo facultativo, fisiologia das raízes e diferentes mecanismos de sobrevivência dos FMA (germinação de esporos, quantidade de inóculo e crescimento micelial).

Maior percentual de colonização das raízes ocorreu durante o período seco (tabela 4). No entanto, há registros divergentes sobre o efeito da estação do ano na colonização. Em uma floresta tropical decídua, 
Allen et al. (1998) registraram que a colonização micorrízica durante a estação chuvosa foi maior do que na estação seca, enquanto em área de caatinga, no Estado de Alagoas, Souza et al. (2003) observaram que os percentuais médios de colonização foram semelhantes nos períodos chuvoso e seco $(\cong 20 \%)$.

Em resumo, maior número de espécies vegetais, de esporos e de propágulos infectivos de FMA foi verificado na área de caatinga nativa preservada, o que indica que a extração de gesso está causando danos ao local, diminuindo a diversidade vegetal e os propágulos de FMA nos solos das áreas exploradas pela mineradora e seus arredores. Práticas que preservem melhor o ambiente são, portanto, altamente recomendadas nas áreas afetadas, considerando a importância desses fungos para o equilíbrio dos ecossistemas onde atuam.

\section{Agradecimentos}

À Dra. Sandra Farto Botelho Trufem, pelas sugestões. À Capes e ao CNPq, pelas bolsas concedidas a A.C.E.S. Mergulhão (Doutorado), L.C. Maia e H.A. Burity (Pesquisa). À Dra. Rita de Cássia Araújo Pereira, da Empresa Pernambucana de Pesquisa Agropecuária (IPA), pela identificação das espécies vegetais e aos revisores anônimos, pelas modificações sugeridas.

\section{Literatura citada}

Abbott, L.K. \& Robson, A.D. 1991. Factors influencing the occurrence of vesicular-arbuscular mycorrhizae. Agriculture, Ecosystems and Environment 35: 121-150.

Allen, E.B., Rincón, E., Allen, M.F., Pérez-Jimenez, A. \& Huante, P. 1998. Disturbance and seasonal dynamics of mycorrhizae in a tropical deciduous forest in Mexico. Biotropica 30: 261-271.

An, Z.Q., Hendrix, J.W., Hershman, D.E. \& Henson, G.T. 1990. Evaluation of the "most probable number" (MPN) and wet-sieving methods for determining soil-borne populations of endogonaceous mycorrhizal fungi. Mycologia 85: 576-581.

Brundrett, M.C. \& Abbott, L.K. 1994. Mycorrhizal fungus propagules in the Jarrah Forest. I. Seasonal study of inoculum levels. New Phytologist 127: 539-546.

Caproni, A.L., Franco, A.A., Berbara, R.L.L., Granha, J.R.D.O., Ribeiro, E.M.S. \& Saggin- Júnior, O.J. 2003. Capacidade infectiva de fungos micorrízicos arbusculares em áreas reflorestadas após mineração de bauxita no Pará. Pesquisa Agropecuária Brasileira 38: 937-945.
Caproni, A.L., Franco, A.A., Berbara, R.L.L., Granha, J.R.D.O. \& Marinho, N.F. 2005. Fungos micorrízicos arbusculares em estéril revegetado com Acacia mangium, após mineração de bauxita. Revista Árvore 29:373-381.

Cavalcanti, A.C. \& Lopes, O.F. 1994. Condições edafoclimáticas da Chapada do Araripe e viabilidade de produção sustentável de culturas. Embrapa, Brasília.

Cochran, W.G. 1950. Estimation of bacterial densities by means of the most probable number. Biometrics 6 : 105-116.

Coelho, F.C., Borges, A.C., Neves, J.C.L., Barros, N.F. \& Muchovej, R.M.C. 1997. Caracterização e incidência de fungos micorrízicos em povoamentos de Eucalyptus grandis e Eucalyptus saligna, nos municípios de Botucatu, São José dos Campos e São Miguel Arcanjo, São Paulo. Revista Árvore 21: 563-573.

Collozi Filho, A. \& Balota, E.L. 1994. Micorrizas Arbusculares. In: M. Hungria \& R.S. Araújo (eds.). Manual de Métodos Empregados em Estudos de Microbiologia Agrícola. Embrapa, Brasília, pp. 383-418.

Day, L.D., Sylvia, D.M. \& Collins, M.E. 1987. Interactions among vesicular-arbuscular mycorrhizae, soil, and landscape positions. Soil Science Society of American Journal 51: 635-639.

Dodd, J., Krikun, J. \& Hass, J. 1983. Relative effectiveness of indigenous populations of vesicular-arbuscular mycorrhizal fungi from four sites in Negev. Israel Journal of Botany 32: 10-21.

Douds Júnior, D.D. 1994. Relationship between hyphal and arbuscular colonization and sporulation in a mycorrhiza of Paspalum notatum Flügge. New Phytologist 126: 233-237.

Feldmann, F. \& Idczak, E. 1992. Inoculum production of vesicular-arbuscular mycorrhizal fungi for use in tropical nurseries. In: J.R. Norris, D.J. Read \& A.K. Varma (eds.). Techniques for Mycorrhizal Research. Methods in Microbiology. Academic Press, London, pp. 799-817.

Gerdemann, J.W. \& Nicolson, T.H. 1963. Spore of mycorrhizal Endogone species extracted from soil by wet sieving and decanting. Transactions of the British Mycological Society 46: 235-244.

Gould, A.B., Hendrix, J.W. \& Ferriss, R.S. 1996. Relationship of mycorrhizal activity to time following reclamation of surface mine land in Western Kentucky. 1. Propagule and spore population densities. Canadian Journal of Botany 74: 247-261.

Jasper, D.A., Abbott, L.K. \& Robson, A.D. 1994. Soil disturbance in native ecosystems - The decline and recovery of infectivity of VA mycorrhizal fungi. In: D.J. Read, D.H. Lewis, A.H. Fitter \& I.J. Alexander (eds.). Mycorrhizas in Ecossystems. CAB International, Cambridge, pp.151-155. 
Jenkins, W.R. 1964. A rapid centrifugal-flotation technique for separating nematodes from soil. Plant Disease Report 48: 692.

Loree, M.A.J. \& Willams, S.E. 1987. Colonization of western wheatgrass (Agropyron smitii Rydb) by vesicular-arbuscular mycorrhizal fungi during the revegetation of a surface mine. New Phytologist 106: 744-753.

Louis, I. \& Lim, G. 1987. Spore density and root colonization of vesicular-arbuscular mycorrhizae in tropical soil. Transactions British Mycological Society 88: 207-212.

Marinho, N.F., Caproni, A.L., Franco, A.A. \& Berbara, R.L.L. 2004. Respostas de Acacia mangium Willd e Sclerolobium paniculatum Vogel a fungos micorrízicos arbusculares nativos provenientes de áreas degradadas pela mineração de bauxita na Amazônia. Acta Botanica Brasilica 18: 141-149.

Marx, D.H. 2000. Stabilizing ecosystems with VAM fungi: significance of VAM fungi in natural plant sucession on disturbed arid and semi-arid lands. PlantHealthCare.com (acesso 02.2000).

Melloni, R., Siqueira, J.O. \& Moreira, F.M.S. 2003. Fungos micorrízicos arbusculares em solos de área de mineração de bauxita em reabilitação. Pesquisa Agropecuária Brasileira 38: 267-276.

Moreira, F.M.S. \& Siqueira, J.O. 2002. Microbiologia e Bioquímica do Solo. Editora UFLA, Lavras.

Phillips, J. M. \& Hayman, D. S. 1970. Improved procedures for clearing roots and staining parasitic and vesiculararbuscular mycorrhizal fungi for rapid assessment of infection. Transactions of the British Mycological Society 55: 158-161.
Sieverding, E. 1991. Vesicular-arbuscular mycorrhiza management in tropical agrosystems. Deutsche Gesellschaft für Technische Zusammenarbeit, Eschborn.

Silva, G.A., Maia, L.C., Silva, F.S.B. \& Lima, P.C.F. 2001 a. Potencial de infectividade de fungos micorrízicos arbusculares oriundos de área de caatinga nativa e degradada por mineração, no Estado da Bahia, Brasil. Revista Brasileira de Botânica 24: 135-143.

Silva, G.A., Santos, B.A., Alves, M.V. \& Maia, L.C. 2001 b. Arbuscular mycorrhiza in species of Commelinidae (Liliopsida) in the State of Pernambuco (Brazil). Acta Botanica Brasilica 15: 155-165.

Silva, G.A., Trufem, S.F.B., Saggin-Júnior, O.J. \& Maia, L.C. 2005. Arbuscular mycorrhizal fungi in a semiarid copper mining area in Brazil. Mycorrhiza 15: 47-53.

Silveira, A.P.D. 1992. Micorrizas. In: E.J.B.N. Cardoso, S.M. Tsai \& M.C.P. Neves (coords.). Microbiologia do Solo. Sociedade Brasileira de Ciência do Solo, Campinas, pp. 257-296.

Siqueira, J.O., Mahmud, A.W. \& Hubbel, D.H. 1986. Comportamento diferenciado de fungos formadores de micorrizas arbusculares em relação à acidez do solo. Revista Brasileira de Ciência do Solo 10: 1-16.

Souza, R.G., Maia, L.C., Sales, M. \& Trufem, S.F.B. 2003. Diversidade e potencial de infectividade de fungos micorrízicos arbusculares em área de caatinga, na Região de Xingó, Estado de Alagoas, Brasil. Revista Brasileira de Botânica 26: 49-60.

STASOFT. 1997. Statistica for Windows. Tulsa.

Zonta, E.P., Machado, A.A. \& Silveira Júnior, P. 1984. Sistema de análise estatística para microcomputadores (SANEST). Departamento de Matemática e Estatística, Pelotas. 